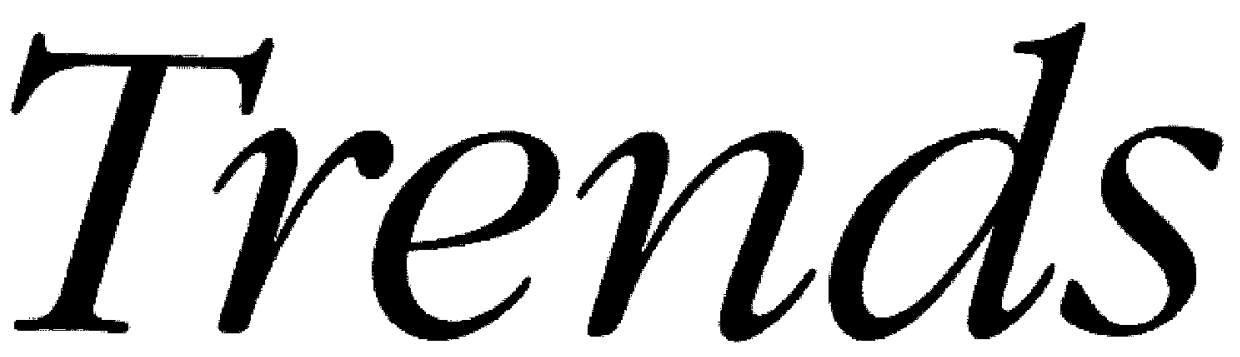

IN LAW LIBRARY MANAGEMENT AND TECHNOLOGY

Edited by Philip C. Berwick • • For academic, firm, corporate, and government law librarians

\title{
A Primer on Digital Object Identifiers for Law Librarians
}

By Benjamin J. KeELE, Reference Librarian, Wolf Law Library, William \& Mary Law School, Williamsburg, Virginia

$\square$ uppose you were to glance at the first page of a scholarly article (say, Boudewijn De Brun, “The Liberal Value of Privacy,” 29 Law \& Philosophy 505 (2010)), you would see a peculiar string of characters: DOI I0.I007/sI0982-010-9067-9. You would find a similar string (doi: I0.I093/jleo/ewnO20) at the bottom-left corner of the first page of Eric Van den Steen, “Disagreement and Allocation of Control," 26 J.L. Econ. \& Org. 385 (2008). These strings are popping up online, too- the online version of an article published in Psychology, Public Policy, and Law and available at http://psycnet.apa.org/journals/law/I6/3/254/ also has this notation: doi: I0.I037/aO0 I 9649. These strings of letters are digital object identifiers (DOIs), and this paper will explain what DOIs are and what they mean to law librarians. 


\section{DOI Functions}

Conceptually, DOIs are pretty simple. At their base, they persistently identify and point to digital objects. Suppose you find an article online that you wish to use. You note its Uniform Resource Locator (URL) so that you can access it again later. You misplace your sticky note and find it two years later while searching your desk for some gum, and are immediately inspired to find that article again. But when you type in the URL, all you find is some page that says "sorry, the page you are looking for it is not here anymore, and we do not know where it went." Sure, if you remember any other bibliographic information, like the article's title or authors, you could do some searching and you may find it again. On the other hand, it has been two years, so you may not remember any more details, and it is also possible that the article has, for whatever reason, just been deleted from any reasonably accessible website.

The problem is that a URL directs your computer to a spot on another computer. If the article is moved from one spot to another spot, like when a website is redesigned or domain names change hands, that URL will not work anymore (unless some other mechanism like a redirect is used). Instead of pointing to a location on a computer, a DOI points to the article itself. So, if you had entered the article's DOI into a resolver, your computer would have found the article, even if it had been moved around the Web in the last two years. If you still could not find it, then either it is actually not online anymore or the article's DOI registration had not been kept current, which is certainly possible, but less likely than a broken URL.

DOIs might look like a random string of characters, but they are actually based on a standardized scheme that is in the final stages of becoming an ISO standard. Every DOI starts with a "IO." prefix to designate it as a DOI. A publisher prefix comes next that indicates the original registrant of the DOI. After the slash is whatever unique set of characters the publisher chooses. Although DOI-based URLs can be used, DOIs themselves are not URLs and do not depend on web browsers, making it more likely they will be usable in postbrowser technology. If a journal is transferred to another publisher, the publisher 
prefix will remain, but since the prefix is not part of any publisher's brand, there is no need to change the DOIs for branding purposes.

Publishers register articles to obtain DOIs. The International DOI Foundation (IDF) designates registration agencies, including CrossRef, the agency that handles DOIs for most scholarly publications. In addition to paying fees for DOIs, publishers commit to providing current metadata about the articles and updating the articles' online locations. When a researcher resolves a DOI, she is sent to the current, publisher-designated version of the article, even if the article has moved from its first online location. Resolving DOIs simply requires adding "http://dx.doi.org/" before the DOI to make it a URL, such as http:// dx.doi.org/I0.I234/vin5a6. DOIs can also be entered into resolvers on CrossRef, http://crossref.org or the IDF's http://www.doi.org websites. Both websites also contain much more detailed technical information on how the DOI System works.

\section{Implications for Law Librarians}

The primary value of DOIs for librarians is that they are persistent identifiers for scholarly articles. A number of studies of the literature in fields such as law, medicine, and science has found that, over time, a large number of URLs break and fail to accurately direct researchers to cited sources. DOIs are more resistant to this breakage; if you see a DOI on an article, then it is probably better to include the DOI or a DOI-based URL in your cite to the article instead of the normal URL because the DOI is more likely, in the long term, to be useful for future researchers. If you see a DOI in a footnote or reference list, using it is a good way to retrieve the article, with the caveat that DOIs lead to the publisherendorsed version, which may or may not be freely accessible. At the very least you will reach a record page that will give you enough bibliographic information to find the article through another source. Some articles that exist in more than one database (JSTOR, Project MUSE, etc.) will have one DOI that gives researchers a choice about which platform to access. The DOI System is one of several persistent identifiers systems, including Archival Resource Keys, Persistent Uniform Resource Locators, Handles, and Uniform Resource Names. While it 


\section{Trends}

is possible that DOIs will eventually become obsolete or be overtaken by another system, thus far the DOI System is the closest to becoming an ISO standard and has been most widely adopted by scholarly publishers.

CrossRef has built additional services on the DOIs it administers. Publishers who obtain DOIs agree to provide metadata about every article and can also provide lists of all cited references in each article. This enables CrossRef to connect cited and citing articles together with their DOIs. An example is an article from the Oxford Journal of Legal Studies (doi: IO.I093/oj1s/22.3.497). Links to articles in CrossRef s system that cite this particular piece can be seen at http://ojls.oxfordjournals.org/cgi/crossref-forward-links/22/3/497. This useful tool helps researchers find additional relevant articles and supports articlelevel impact metrics. Shepard's, KeyCite, and HeinOnline's ScholarCheck connect citing articles within each vendor's database, but these systems require a subscription to access and are not interoperable with each other or with DOIs that link non-legal articles.

CrossRef s linking system relies on the participation of many publishers. A small study I conducted, available at http://works.bepress.com/ aallcallforpapers $/ 65 /$, indicated that very few law reviews assign DOIs to their articles or include DOIs in their footnotes. While this limits the usefulness of DOIs for legal researchers, DOIs are still valuable for accessing and citing articles in other disciplines. As legal scholarship becomes more interdisciplinary and more law professors have scholarly interests in the intersection of law with other academic fields, law librarians will probably encounter more articles with DOIs.

Should law journals use DOIs in their footnotes or assign DOIs to their own articles? Including DOIs or DOI-based URLs in footnotes when possible will increase the reliability of references to online scholarly articles, but it will also require some additional work by journal staff. My study details how I found DOIs using bibliographic data for articles. Although the Bluebook encourages citing to the most permanent and reliable version of a source, it does not mention DOIs, which significantly reduces law journal editors' incentive to use them. The Publication Manual of the American Psychological Association, on the other hand, calls for including DOIs in references. This does not itself mean law journals should follow the APA, but rather shows that other disciplines, whose 
articles legal scholars sometimes read and cite, assign DOIs to their articles and cite them accordingly. Law librarians can use DOIs to assist patrons who are conducting research in non-legal fields.

Assigning DOIs to law journal articles will require more than adding a few steps to the cite-checking process. Obtaining DOIs costs money, including annual CrossRef membership dues and twenty-five cents per DOI. Aside from just finding money in the budget, a greater obstacle is fulfilling the commitment to provide article metadata and reference lists in the proper XML format. For journals with frequent staff turnover, cultivating the tools and expertise to complete this task can be a major challenge. The most promising solution seems to be delegating this work to a vendor that serves a number of law journals. Just as there is a small set of printers that specialize in law journal production, perhaps some vendors could handle DOI registration and management for dozens, or even hundreds, of journals. Another option would be journals forming a consortium to pool their resources. JSTOR assigns DOIs to articles from some of the few law journals it contains, including the Harvard Law Review, Duke Law Journal, and Yale Law Joumal, so it clearly is possible for a vendor to register DOIs on a journal's behalf. A list of titles that participate in CrossRef is available at http://www.crossref.org/titleList/.

Whether to assign DOIs to articles must be decided on the basis of each journal's resources and business model. While I think DOIs would be useful for all journals, and the more journals that use DOIs the more valuable the referencelinking system becomes, three types of journals are the best candidates. Onlineonly journals could benefit from DOIs because their format does not lend itself well to the traditional, paper-based, volume-journal-first page citation system, and because if an article's URL breaks, access and discoverability are more likely to be seriously limited. Law journals with an interdisciplinary focus are more likely to cite and be cited by non-legal journals that use DOIs, so adopting DOIs would more fully connect legal scholarship with other relevant non-legal work. Finally, very prestigious, high-impact journals are also more likely to be cited outside the legal academy and thus should take measures to ensure long-term access. 


\section{Trends}

\section{Conclusion}

While DOIs have taken a role in article linking and references in non-legal academic fields, they have thus far not been paid much attention by legal researchers. By maintaining at least basic familiarity with the function and use of DOIs, law librarians will be better equipped to assist researchers with interdisciplinary research, to advise professors and journals if they start thinking about using DOIs, and to improve the reliability and usefulness of scholarly citations.

Benjamin J. Keele, Reference Librarian, Wolf Law Library, William \& Mary Law School, Williamsburg,Virginia; email:bjkeele@wm.edu. Copyright2010by Benjamin J.Keele. This article is distributed under the terms of the Creative Commons Attribution License http:/ / creativecommons,org/licenses/by/3.0, which permits untestricted use, distribution, and reproduction in any medium, provided the original author and source are credited.

Trends is published by

William S. Fein \& Co, Inc.

I285 Main St. / Buffalo, NY 14209

Phone: (800) 828-757 I; (716) 882-2600

Fax: (716) 883-8100

Email:mail@wshein.com

ISSN 0893-6773

The complete collection of articles published

in Trends is available in HeinOnline

(http://heinonline.org)
Editor: Philip C. Berwick, Associate Dean

for Information Resources

Washington University School of Law

Law Library

Campus Box I I7 I

St. Louis, MO 63130

Phone: (314) 935-4042; Fax: (314) 935-7125

Email:<BERWICK@WULAW.WUSTL.EDU>

(C) 2010 by William S. Hein \& Co., Inc. 